\title{
Magnetic and Magnetotransport Properties of Magnetite/Co-Ferrite/Magnetite Trilayers
}

\author{
Y.F. $\mathrm{CHEN}^{a}$ AND M. ZIESE ${ }^{b}$ \\ ${ }^{a}$ State Key Laboratory of Electronic Thin Films and Integrated Devices \\ University of Electronic Science and Technology of China \\ Chengdu 610054, People's Republic of China \\ ${ }^{b}$ Division of Superconductivity and Magnetism, University of Leipzig \\ D-04103 Leipzig, Germany
}

\begin{abstract}
The magnetic and magnetotransport properties of $\mathrm{Fe}_{3} \mathrm{O}_{4} / \mathrm{CoFe}_{2} \mathrm{O}_{4} / \mathrm{Fe}_{3} \mathrm{O}_{4}$ trilayers were investigated. The magnetization measurements indicate significant Co diffusion into the bottom magnetite layer. At lower temperatures the magnetoresistance measurements show two clearly separated maxima due to contributions from magnetically hard and soft layers. A tunneling effect was not observed. Comparison of full and minor resistance hysteresis loops did not reveal any significant exchange coupling between the layers, presumably due to diffuse interfaces.
\end{abstract}

PACS numbers: 72.25.-b, 72.25.Mk, 75.70.-i

\section{Introduction}

Recently the spin-filter diode [1] has been developed as an alternative to the magnetic tunneling junction $[2,3]$. In this device concept the tunneling barrier plays an active role in spin-polarizing the current passing between a ferromagnetic electrode and a non-ferromagnetic counter-electrode. Since the spin direction is conserved in direct quantum mechanical tunneling, the resistance of the spin-filter diode shows a strong dependence on the relative orientation of the magnetization vectors in the ferromagnetic electrode and the barrier $[1,4-6]$. In this work a combination of conventional tunneling junction and spin-filter diode is investigated in the all-oxide $\mathrm{Fe}_{3} \mathrm{O}_{4} / \mathrm{CoFe}_{2} \mathrm{O}_{4} / \mathrm{Fe}_{3} \mathrm{O}_{4}$ system. The motivation in choosing ferrites lied in their predicted high spin-polarization $[7,8]$ and the high Curie temperatures of

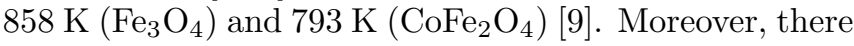
has been considerable work on ferrite heterostructures [10-12] and Co-ferrite has already been used as spin-filter in a $\mathrm{La}_{0.7} \mathrm{Sr}_{0.3} \mathrm{MnO}_{3} / \mathrm{CoF}_{2} \mathrm{O}_{4} / \mathrm{Nb}(0.5 \%): \mathrm{SrTiO}_{3}$ heterostructure [13].

\section{Experimental}

$\mathrm{Fe}_{3} \mathrm{O}_{4} / \mathrm{CoFe}_{2} \mathrm{O}_{4} / \mathrm{Fe}_{3} \mathrm{O}_{4} \quad$ (FEO/CFO/FEO) samples were fabricated by pulsed laser deposition from stoichiometric $\mathrm{Fe}_{3} \mathrm{O}_{4}$ and $\mathrm{CoFe}_{2} \mathrm{O}_{4}$ targets onto $\mathrm{MgO}$ (001) substrates at $450^{\circ} \mathrm{C}$ in an oxygen partial pressure of $10^{-5}$ mbar. Just after the deposition of the bottom $\mathrm{Fe}_{3} \mathrm{O}_{4}$ layer, a mask was used to cover half of the substrate, and subsequently the $\mathrm{CoFe}_{2} \mathrm{O}_{4}$ spacer layer and the top $\mathrm{Fe}_{3} \mathrm{O}_{4}$ electrode were deposited. X-ray diffractometry measurements showed epitaxial growth of the magnetite layers, whereas the Co-ferrite spacer layer could not be resolved.

Here data on a sample with layer structure of $15 \mathrm{~nm}$ (top FEO)/8 nm (CFO)/60 nm (bottom FEO) are reported. Electrical contacts were made with silver paste and copper wires on the bottom and top $\mathrm{Fe}_{3} \mathrm{O}_{4}$ electrodes, respectively. The magnetoresistance was measured in a four-point out-of-plane configuration. Magnetization measurements were performed with a SQUID magnetometer. The diamagnetic contribution of the substrate was subtracted from the data.

\section{Results and discussion}

Figure 1 shows the magnetic characterization of the sample. The most striking feature is revealed by the lower panel showing magnetization hysteresis loops. From the structural data it is expected that about $10 \%$ of the sample contain the magnetically hard Co-ferrite phase. The magnetization data, however, show that this is not so, but that about $65 \%$ of the sample are magnetically hard. This can only be due to Co-diffusion from the Co-ferrite spacer layer preferably into the bottom electrode layer $[14,15]$. Since this is a priori not unexpected, we have never observed this before in our work on $\mathrm{Fe}_{3} \mathrm{O}_{4} / \mathrm{Co}_{0.16} \mathrm{Fe}_{2.84} \mathrm{O}_{4}$ bilayers [16]. Obviously the diffusion rate in the present case is considerably larger due to the high Co-concentration in the $\mathrm{CoFe}_{2} \mathrm{O}_{4}$ layer. In Fig. 1a the magnetization is shown as a function of tem- 
perature. Below $150 \mathrm{~K}$ there is a significant difference between the magnetization curves measured in magnetic fields of $0.3 \mathrm{~T}$ and $1.0 \mathrm{~T}$ due to the temperature dependence of the coercive field of the hard layer. At about $100 \mathrm{~K}$ a small step-like anomaly can be seen in the magnetization curves; this is more clearly observed in the inset in the derivative $\mathrm{d} M / \mathrm{d} T$-curves. This might either be due to the Verwey transition or to the crossing of applied field and coercive field in the hard layer, $H_{\mathrm{c}}(T)=H$. Since the anomaly - even if very faint - is still present in an applied field of $1 \mathrm{~T}$ with the sample already being in technical saturation, it is more likely to be caused by the Verwey transition of the top magnetite electrode.

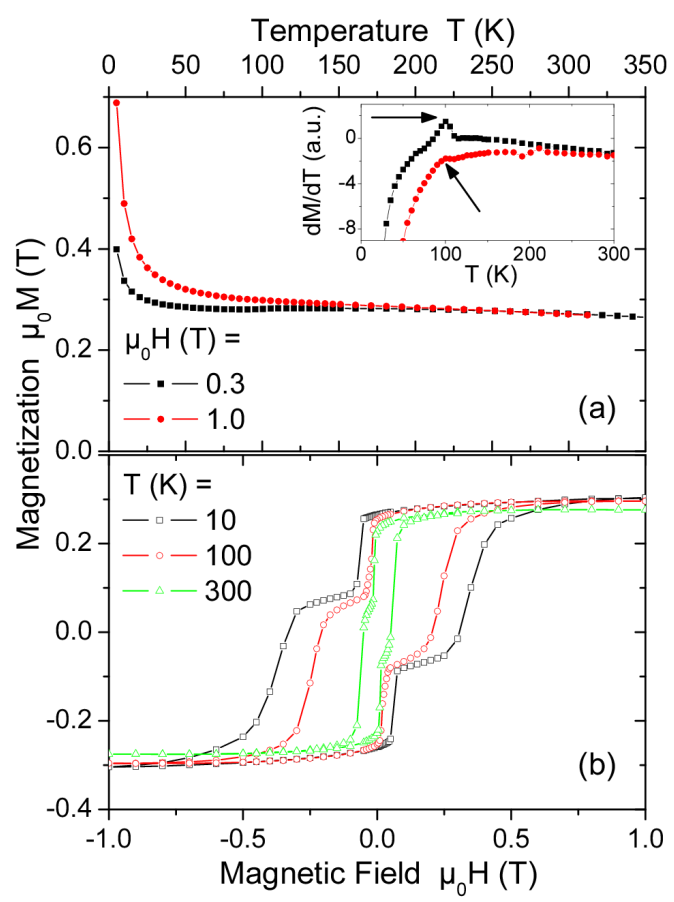

Fig. 1. (a) Magnetization of the sample as a function of temperature for applied magnetic fields of 0.3 and $1.0 \mathrm{~T}$. The inset shows the temperature derivative of the magnetization. The arrows indicate weak anomalies in the derivative at $110 \mathrm{~K}$. (b) Magnetization hysteresis loops measured at 10, 100 and $300 \mathrm{~K}$.

Current-voltage characteristics measured at temperatures between $80 \mathrm{~K}$ and $300 \mathrm{~K}$ were linear. This precludes the observation of a tunneling current and tunneling magnetoresistance. In view of the strong $\mathrm{Co} / \mathrm{Fe}-$ -interdiffusion, this is not surprising. The resistivity of the bottom electrode was measured by a four point method. The resistivity shows clearly insulating behavior without any obvious resistivity jump that would reveal the Verwey transition.

The magnetoresistance (MR) MR $=[R(H)-R(0)] /$ $R(0)$ measured in an applied field parallel to the sample is shown in Fig. 2a for a temperature of $110 \mathrm{~K}$. The MR clearly shows the typical butterfly contributions from both the magnetically soft and the hard phase. The max- ima and shoulders of the MR occur at the coercive fields as indicated by the arrows. The MR is comparatively small and is certainly due both to anisotropic magnetoresistance and, since it shows a considerable high field slope, to grain-boundary magnetoresistance [16]. The general shape of the MR-curves does not change with temperature, the MR-value at $0.6 \mathrm{~T}$ decreases to $-0.7 \%$ at $300 \mathrm{~K}$.

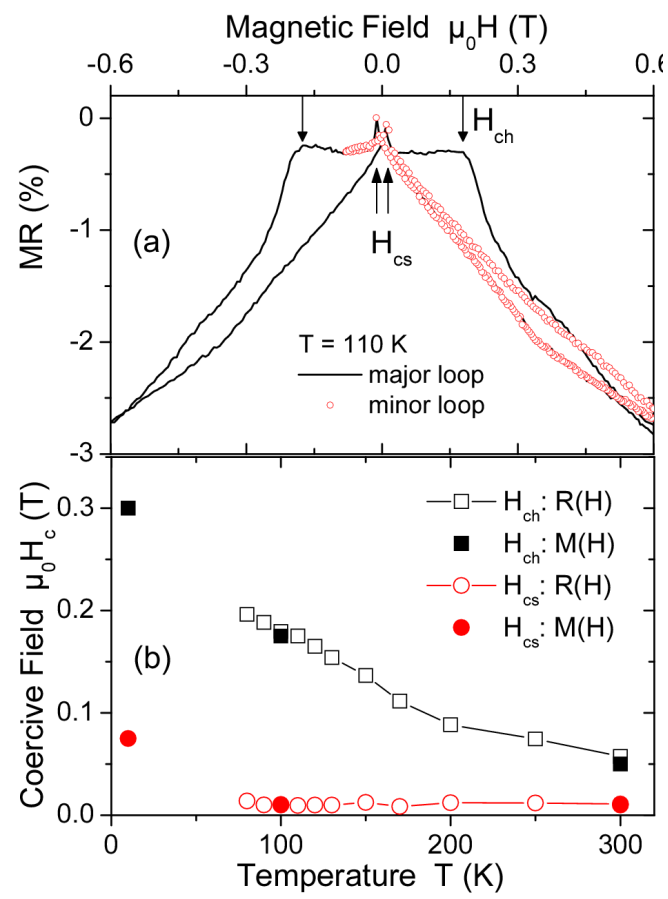

Fig. 2. (a) Full and minor resistivity hysteresis loops measured at $110 \mathrm{~K}$. The magnetic field was applied parallel to the sample. The arrows mark the coercive fields of the magnetically soft $\left(H_{\mathrm{cs}}\right)$ and hard $\left(H_{\mathrm{ch}}\right)$ phases. (b) Coercive fields of the magnetically soft and hard phases as determined from resistivity and magnetization.

The magnetic coupling between the magnetically soft and hard layers was studied by magnetoresistance measurements. Besides the full magnetoresistance loop Fig. 2a also shows a minor loop. In the minor loop the magnetoresistance shoulder for positive magnetic fields is absent, since the hard layer was not reversed. However, no significant shift of the magnetoresistance maxima of the soft layer occurring at $H_{\mathrm{cs}}$ could be detected. This indicates that the magnetic coupling between the hard and the soft layers is weak. This is in contrast to the results on $\mathrm{Fe}_{3} \mathrm{O}_{4} / \mathrm{Co}_{0.16} \mathrm{Fe}_{2.84} \mathrm{O}_{4}$ bilayers [17], which showed a strong coupling between the two layers. We believe that this is due to the absence of a sharp interface in the present samples due to the strong interdiffusion.

The coercive fields of the magnetically hard and soft phases were determined from the magnetization hysteresis loops and the maxima in the magnetoresistance hysteresis loops, respectively. The coercivity of the magnetite layer shows the generic temperature depen- 
dence [18] being almost temperature independent between $100 \mathrm{~K}$ and $300 \mathrm{~K}$ and rising below $100 \mathrm{~K}$ due to the change of the magnetocrystalline constants at the Verwey transition, see Fig. 2b. The Co-doped magnetite layer shows a continuous rise in coercivity on cooling from $300 \mathrm{~K}$ down to $10 \mathrm{~K}$ in qualitative agreement with the results on $\mathrm{Co}_{0.16} \mathrm{Fe}_{2.84} \mathrm{O}_{4}$ layers [17].

\section{Conclusions}

$\mathrm{Fe}_{3} \mathrm{O}_{4} / \mathrm{CoFe}_{2} \mathrm{O}_{4} / \mathrm{Fe}_{3} \mathrm{O}_{4}$ samples were fabricated by pulsed laser deposition and their magnetic and magnetotransport properties were studied. The magnetization measurements indicate strong $\mathrm{Co} / \mathrm{Fe}$-interdiffusion with the formation of a large fraction of a magnetically hard phase, i.e. Co-doped magnetite, in these samples. Tunneling conduction was absent. The magnetoresistance contained contributions from both phases due to anisotropic and grain-boundary magnetoresistance. The magnetic coupling between the magnetically hard and soft phases was weak, presumably due to diffuse interfaces. The coercive fields determined from magnetization and magnetoresistance hysteresis loops were in good agreement.

\section{Acknowledgments}

This work was supported by the DFG within the Collaborative Research Center (SFB 762) "Functionality of Oxide Interfaces".

\section{References}

[1] P. LeClair, J.K. Ha, H.J.M. Swagten, J.T. Kohlhepp, C.H. van de Vin, W.J.M. de Jonge, Appl. Phys. Lett. 80, 625 (2002).

[2] M. Julliere, Phys. Lett. A 54, 225 (1975).

[3] A. Gupta, J.Z. Sun, J. Magn. Magn. Mater. 200, 24 (1999).
[4] M. Gajek, M. Bibes, A. Barthélémy, K. Bouzehouane, S. Fusil, M. Varela, J. Fontcuberta, A. Fert, Phys. Rev. B 72, 020406(R) (2005).

[5] U. Lüders, M. Bibes, K. Bouzehouane, E. Jacquet, J.-P. Contour, S. Fusil, J.-F. Bobo, J. Fontcuberta, A. Barthélémy, A. Fert, Appl. Phys. Lett. 88, 082505 (2006).

[6] U. Lüders, A. Barthélémy, M. Bibes, K. Bouzehouane, S. Fusil, E. Jacquet, J.-P. Contour, J.-F. Bobo, J. Fontcuberta, A. Fert, Adv. Mater. 18, 1733 (2006).

[7] A. Yanase, N. Hamada, J. Phys. Soc. Japan 68, 1607 (1999).

[8] C. Srinitiwarawong, G. Gehring, J. Phys., Condens. Matter 13, 7987 (2001).

[9] A.H. Morrish, The Physical Principles of Magnetism, IEEE Press, New York 2001.

[10] P.A.A. van der Heijden, P.J.H. Bloemen, J.M. Metselaar, R.M. Wolf, J.M. Gaines, J.T.W.M. van Eemeren, P.J. van der Zaag, W.J.M. de Jonge, Phys. Rev. B 55, 11569 (1997).

[11] P.J. van der Zaag, P.J.H. Bloemen, J.M. Gaines, R.M. Wolf, P.A.A. van der Heijden, R.J.M. van de Veerdonk, W.J.M. de Jonge, J. Magn. Magn. Mater. 211, 301 (2000).

[12] E. Snoeck, Ch. Gatel, R. Serra, G. BenAssayag, J.-B. Moussy, A.M. Bataille, M. Pannetier, M. Gautier-Soyer, Phys. Rev. B 73, 104434 (2006).

[13] Y.F. Chen, M. Ziese, Phys. Rev. B 76, 014426 (2007).

[14] R. Dieckmann, Habilitationsschrift, Fachbereich Chemie der Universität Hannover, Hannover 1983.

[15] R. Dieckmann, T.O. Mason, J. Hodge, H. Schmalzried, Ber. Bunsenges. Phys. Chem. 82, 778 (1978).

[16] M. Ziese, R. Höhne, H.-C. Semmelhack, H. Reckentin, N.H. Hong, P. Esquinazi, Eur. Phys. J. B 28, 415 (2002).

[17] M. Ziese, R. Höhne, A. Bollero, H.-C. Semmelhack, P. Esquinazi, K. Zimmer, Eur. Phys. J. B 45, 223 (2005).

[18] A. Bollero, M. Ziese, R. Höhne, H.-C. Semmelhack, U. Köhler, A. Setzer, P. Esquinazi, J. Magn. Magn. Mater. 285, 279 (2005). 